Cite this: Phys. Chem. Chem. Phys., 2011, 13, 9676-9684

\title{
Five isomers of monomeric cytosine and their interconversions induced by tunable UV laser light $\uparrow$
}

\author{
Leszek Lapinski, ${ }^{a}$ Igor Reva, $^{b}$ Maciej J. Nowak $^{* a}$ and Rui Fausto ${ }^{b}$ \\ Received 7th December 2010, Accepted 10th March 2011 \\ DOI: $10.1039 / \mathrm{c0cp02812f}$
}

Photoisomerization processes involving five isomers of cytosine were induced by narrowband tunable UV irradiation of matrix-isolated monomers of the compound. Irradiation of an argon matrix containing cytosine monomers with UV $\lambda=313 \mathrm{~nm}$ laser light resulted in syn $\leftrightarrow$ anti photoisomerizations between the two imino-oxo forms, whereas the substantially more populated amino-hydroxy and amino-oxo forms stayed intact. Subsequent irradiation with shorter-wavelength UV $\lambda=311 \mathrm{~nm}$ laser light led to two concomitant phototautomeric processes consuming the amino-oxo isomer: (i) an oxo $\rightarrow$ hydroxy hydrogen-atom transfer photoprocess converting the amino-oxo form into the amino-hydroxy tautomer; (ii) amino $\rightarrow$ imino hydrogen-atom transfer converting the amino-oxo form into the imino-oxo isomers. The UV-induced phototransformations, together with mutual conversions of the two amino-hydroxy conformers induced by irradiation with narrowband NIR light, allowed positive detection and identification of the five isomeric forms of monomeric cytosine. This is the first experimental observation of all five low-energy isomers of cytosine.

\section{Introduction}

In the recent decade, photophysics of cytosine monomers in the gas phase was intensively studied using modern experimental ${ }^{1,2}$ as well as theoretical ${ }^{3}$ methods. Experimental investigations on picosecond and subpicosecond excited-state dynamics revealed multiexponential decay following the UV excitation of cytosine. Two dominating components of this decay are characterized by subpicosecond $160 \mathrm{fs}$ and picosecond 1.9 ps lifetimes. ${ }^{1}$ Several mechanisms of the ultrafast decay of electronically excited cytosine were conceived ${ }^{3}$ and supported by the state-of-the-art calculations of excited-state potential energy surfaces. The general picture of very effective intramolecular quenching of the cytosine excited state(s), prohibiting formation of structurally modified photoproducts, stems from both experimental and theoretical works. In line with these conclusions, no products of unimolecular photochemistry of cytosine were proposed. Moreover, the question of the structure, actually adopted by cytosine molecules in the gas phase prior to any excitation, was mostly disregarded in these studies.

${ }^{a}$ Institute of Physics, Polish Academy of Sciences, Al. Lotnikow 32/46, 02-668 Warsaw, Poland.E-mail: mjnowak@ifpan.edu.pl

${ }^{b}$ Department of Chemistry, University of Coimbra, 3004-535 Coimbra, Portugal

$\dagger$ Electronic supplementary information (ESI) available: The effects of irradiation of matrix-isolated cytosine with NIR and UV $(\lambda=300 \mathrm{~nm})$ light (Fig. S1 and S2); spectral indications of the AO $\rightarrow$ AH phototautomeric reaction (Fig. S3); assignment of the observed IR bands to AH1, AH2, AO, IO1 or IO2 forms (Fig. S4 and Table S1); IR bands due to IO2 form growing upon UV $(\lambda=300 \mathrm{~nm})$ irradiation (Fig. S5). See DOI: $10.1039 / \mathrm{c} 0 \mathrm{cp} 02812 \mathrm{f}$
On the other hand, numerous theoretical ab initio calculations on relative energies of cytosine tautomers were carried out in recent years. The results of the contemporary quantum chemical calculations $^{4,5}$ predict a consistent (and probably correct) energy ordering of cytosine isomers. According to these calculations, carried out at the CCSD(T) or QCISD(T) levels, the most stable tautomer of monomeric cytosine should be the amino-hydroxy (AH) form. Of the two AH conformers (differing by rotation of the hydroxyl group by $c a .180^{\circ}$, see Scheme 1), AH1 was theoretically predicted to be more stable by $2.9-3.1 \mathrm{~kJ} \mathrm{~mol}^{-1}$ than AH2. The energy of the amino-oxo (AO) form was theoretically computed to be higher by $4.9-6.3 \mathrm{~kJ} \mathrm{~mol}^{-1}$ than<smiles>Nc1ccn(C=O)c(=O)n1</smiles><smiles>N=c1cc[nH]c(=O)[nH]1</smiles>

IO 1<smiles>Nc1ccnc(O)n1</smiles>

AH 1<smiles>N=c1cc[nH]c(=O)[nH]1</smiles>

IO 2<smiles>Nc1nccc(O)n1</smiles>

AH 2
Scheme 1 Structures of the lowest-energy isomeric forms of cytosine. 
the energy of the most stable AH1 isomer. As far as the imino-oxo forms are concerned, the relative energies of IO1 and $\mathbf{I O 2}$ should be $c a$. 7 and $12 \mathrm{~kJ} \mathrm{~mol}^{-1}$, with respect to AH1. Hence, for cytosine in the gas phase, together with the dominating AH1 and AH2 isomers, also AO and IO1 forms should be significantly populated. The relative population of IO2 should be much lower, and all the remaining forms of cytosine (whose relative energies are much higher) should not be populated at all.

Experimental studies on relative populations of cytosine isomers in the gas phase are by far less abundant. The most populated $\mathbf{A H}$ and $\mathbf{A O}$ forms were experimentally found using microwave $^{6}$ and photoelectron ${ }^{7}$ techniques. Forms AH1, AH2 and AO were identified for cytosine monomers trapped in helium nanodroplets at $0.37 \mathrm{~K}$. ${ }^{8}$ So far, no unquestionable experimental evidence of the imino-oxo IO forms of gaseous cytosine has been provided.

As revealed by previous investigations, ${ }^{9,10}$ monomers of cytosine, in different isomeric forms, can be effectively trapped from the gas phase into low-temperature matrices. In our recent study, ${ }^{9}$ matrix-isolated cytosine was irradiated with narrowband near infrared (NIR) light. The dominating amino-hydroxy AH1 and $\mathbf{A H 2}$ isomers were found to convert into each other upon NIR irradiation at 7013 or at $7034 \mathrm{~cm}^{-1}$. On the basis of this effect, two sets of IR bands were assigned to AH1 and AH2. Together with the IR spectral features attributed to AH1 and AH2, a set of bands not affected by any NIR irradiation was observed. This demonstrated that, alongside AH1 and AH2, other isomers of cytosine were trapped in solid argon.

Effects of UV irradiation can be used to differentiate between the isomeric forms of cytosine codeposited in the same matrix. In previous investigations, ${ }^{10,11}$ monomers of cytosine isolated in $\mathrm{Ar}$ or $\mathrm{N}_{2}$ matrices were irradiated with broadband UV light. High-pressure xenon or mercury lamps, fitted with appropriate cutoff filters, were used for this purpose. This approach allowed unequivocal identification of $\mathbf{A H}$ and AO forms of matrix-isolated cytosine. However, the intrinsic limitations of broadband UV irradiation did not allow a very detailed analysis of the observed photoeffects. In particular, it was not possible to separately observe the photoreactions of the minor IO forms of the compound.

In the current study, matrix-isolated cytosine monomers were excited at a series of chosen wavelengths using tunable, narrowband UV laser light. Such an approach permitted us to obtain a much clearer picture of the UV-induced transformations of monomeric cytosine, including separate observation of phototransformations of $\mathbf{I O}$ and $\mathbf{A O}$ forms. This, in turn, allowed a significantly more conclusive analysis of the experimental results, with special emphasis devoted to the photochemistry of the minor, imino-oxo IO forms.

\section{Methods}

\subsection{Experimental section}

The crystalline sample of cytosine (purity 99\%) used in the present study was a commercial product supplied by Sigma. In order to prepare Ar matrices containing isolated monomers of cytosine, a solid sample of the compound was heated (to $c a .495 \mathrm{~K}$ ) in a miniature glass oven placed in the vacuum chamber of a helium-cooled cryostat. Similarly to the previous studies, ${ }^{6,10,11}$ no signs of thermal decomposition were observed for cytosine sublimating at $495 \mathrm{~K}$. Vapors of cytosine were deposited together with large excess of argon (purity N6.0, supplied by Air Liquide) onto a CsI window cooled to $12 \mathrm{~K}$. The IR spectra were recorded in the $4000-400 \mathrm{~cm}^{-1}$ range, with $0.5 \mathrm{~cm}^{-1}$ resolution, using a Thermo Nicolet 670 FTIR spectrometer equipped with a KBr beam splitter and a DTGS detector. Matrices were irradiated with the frequency doubled signal beam of the Quanta-Ray MOPO-SL pulsed (10 ns) optical parametric oscillator $\left(\mathrm{FWHM} \approx 0.2 \mathrm{~cm}^{-1}\right.$, repetition rate $10 \mathrm{~Hz}$, pulse energy $\approx 1.0 \mathrm{~mJ}$ ) pumped with a pulsed Nd:YAG laser.

\subsection{Computational section}

The geometries of the five lowest-energy isomeric forms of cytosine (see the structures presented in Scheme 1) were fully optimized using the density functional method DFT(B3LYP) with Becke's three-parameter exchange functional ${ }^{12}$ and the Lee, Yang, Parr correlation functional. ${ }^{13}$ The $6-31++G(d, p)$ basis set was applied in these calculations. At the optimized geometries, the harmonic vibrational frequencies and IR intensities were calculated at the same DFT(B3LYP)/6-31++G(d,p) level. The computed harmonic vibrational wavenumbers were scaled down by a single factor of 0.978 . All the calculations were performed with the Gaussian 03 program. ${ }^{14}$

\section{Results and discussion}

Monomers of cytosine were deposited into low-temperature Ar matrices. The infrared spectra of these matrices were virtually the same as those recorded in the previous works. ${ }^{10,11}$ The quality of the spectra obtained in the current experiments was somewhat better, due to the higher sensitivity and resolution of the used spectrometer.

In the present work, matrix-isolated cytosine was irradiated with UV light of a frequency doubled signal beam of the optical parametric oscillator $\left(\mathrm{FWHM} \approx 0.2 \mathrm{~cm}^{-1}\right.$ ). The preliminary choice of wavelengths of the applied UV light was guided by the absorption spectra of isolated cytosine monomers, recorded by Nir et al. ${ }^{15}$ using the REMPI technique. In the long wavelength region of the REMPI spectra, medium-strong absorptions were observed at 313-314 nm. These absorptions were assigned to AO form of the compound. At longer wavelengths (315-316 nm) only significantly weaker bands were found. Those bands were tentatively assigned to other isomers, probably to IO. Absorptions due to the $\mathbf{A H}$ tautomer appeared at much shorter wavelengths (ca. $275 \mathrm{~nm})$.

\subsection{Syn $\leftrightarrow$ anti photoisomerization in the imino-oxo forms}

The UV irradiations of matrix-isolated cytosine carried out in the present study started at wavelength $320 \mathrm{~nm}$. After each irradiation the sample was monitored by recording an infrared spectrum. No photoreactions occurred in matrices irradiated at wavelengths $320-316 \mathrm{~nm}$. The first phototransformations were observed for $\lambda=314 \mathrm{~nm}$, though the effects of irradiation at this wavelength were very slight. More pronounced 


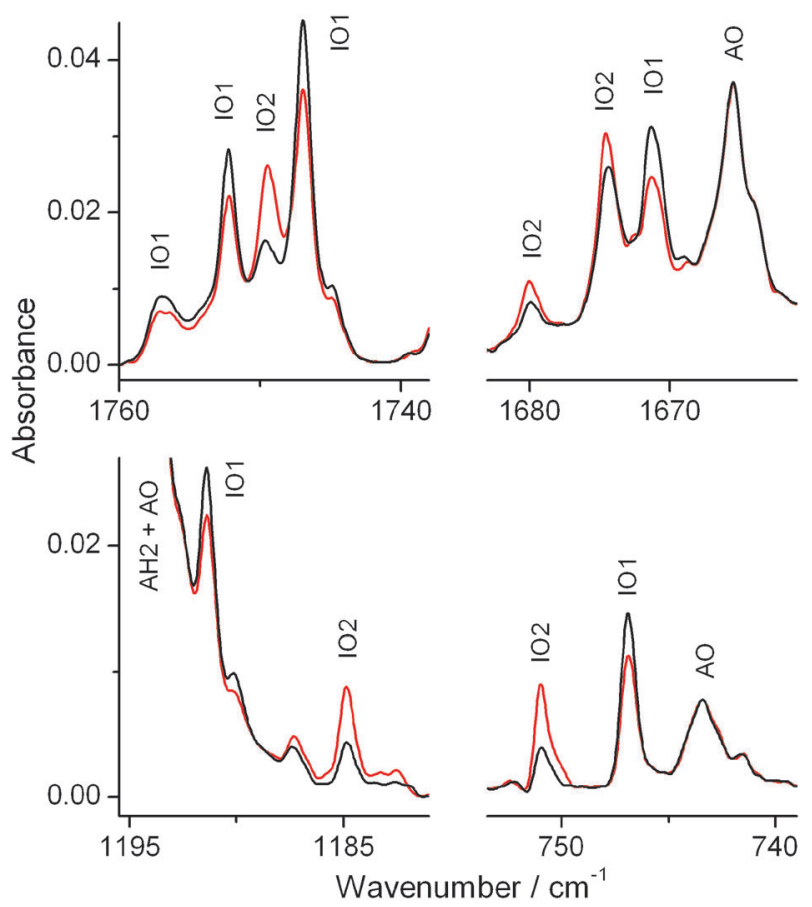

Fig. $1 S y n \leftrightarrow$ anti isomerization of the imino-oxo forms of cytosine induced by UV $(\lambda=312 \mathrm{~nm})$ irradiation with monochromatic laser light. Fragments of the infrared spectrum of cytosine monomers isolated in an Ar matrix: (black) recorded after deposition of the matrix; (red) recorded after irradiation at $312 \mathrm{~nm}$.

changes in the IR spectra occurred upon irradiation at 313 and $312 \mathrm{~nm}$. The changes induced by irradiations at 313 and $312 \mathrm{~nm}$ concerned only two sets of low- or very low-intensity bands. All of those bands were already affected by UV $(\lambda=314 \mathrm{~nm})$ light. Upon irradiations at 314,313 or $312 \mathrm{~nm}$, one set of bands decreased in intensity, whereas the intensities of the bands belonging to the other set increased (see Fig. 1). On the other hand, irradiations at these wavelengths did not affect any intense bands in the IR spectrum. This indicated that the substrate of the photoreaction as well as the photoproduct must be minor isomers of cytosine. The obvious candidates for these isomers are the imino-oxo IO1 and IO2 forms.

Taking into account the theoretically predicted relative energies of the imino-oxo forms, IO1 should be significantly more populated than IO2 in a matrix before any irradiation. ${ }^{5,16}$ Because the form being consumed upon UV $(\lambda=314,313$ or $312 \mathrm{~nm}$ ) irradiation is more populated in a freshly deposited matrix, it can be tentatively assigned to IO1. Consequently, the form being photoproduced upon such irradiation can be assigned to IO2. The photoreaction transforming one of the imino-oxo forms into the other is a syn $\leftrightarrow$ anti photoisomerization or, in other words, a flip of the hydrogen atom of the imino group (see Scheme 2). A similar photoisomerization reaction was previously observed for the imino-oxo isomers of matrix-isolated 1-methylcytosine. ${ }^{17}$

The positions of the IR bands in the spectrum of the substrate and of the product of the photoreaction induced by irradiation of cytosine at 314,313 or $312 \mathrm{~nm}$ confirm the above interpretation. In the $1800-1600 \mathrm{~cm}^{-1}$ spectral range, where the bands due to stretching vibrations of the $\mathrm{C}=\mathrm{O}$,

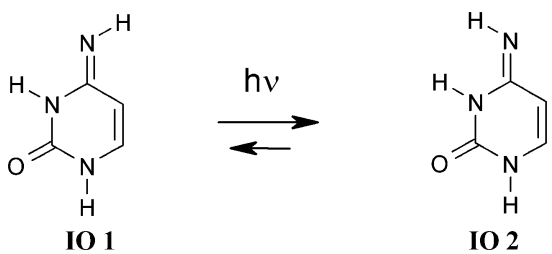

Scheme 2 UV-induced isomerization transforming one of the iminooxo forms of cytosine into the other.

$\mathrm{C}=\mathrm{N}$ and $\mathrm{C}=\mathrm{C}$ double bonds can be expected, the IR absorptions decreasing upon UV $(\lambda=314,313$ or $312 \mathrm{~nm})$ irradiations appear at $1757 / 1752 / 1747$ and at $1671 \mathrm{~cm}^{-1}$, whereas those increasing in intensity were found at 1750 and $1675 \mathrm{~cm}^{-1}$. In the spectra of both forms involved in the observed photoconversion, the bands at $\sim 1750 \mathrm{~cm}^{-1}$ should originate from the stretching vibrations of the carbonyl group $(\nu \mathrm{C}=\mathrm{O})$. Such a wavenumber is higher by $\sim 30 \mathrm{~cm}^{-1}$ than that of the IR band due to the $\nu \mathrm{C}=\mathrm{O}$ vibration in the $\mathbf{A O}$ form of cytosine. In the experimental IR spectrum, this last band was found at $1720 / 1717 \mathrm{~cm}^{-1}$. The bands due to $\nu \mathrm{C}=\mathrm{O}$ vibrations were theoretically predicted at 1762, 1758 and $1735 \mathrm{~cm}^{-1}$ for IO1, IO2 and AO, respectively. Hence, according to the calculations, the $\nu \mathrm{C}=\mathrm{O}$ bands in the spectra of IO1 and IO2 should partly overlap and should appear at wavenumbers higher by some $25 \mathrm{~cm}^{-1}$, with respect to the $\nu \mathrm{C}=\mathrm{O}$ band of $\mathbf{A O}$. This theoretical prediction is in agreement with the experimental picture and supports the assignment of IO1 and IO2 to the forms involved in the photoconversion induced by UV irradiation at $314-312 \mathrm{~nm}$.

The low-intensity bands increasing or decreasing upon irradiation at 314-312 nm were observed throughout the whole IR spectrum of cytosine. Many of them overlap with much stronger bands of $\mathbf{A H}$ and AO forms. The clearly observed spectral features of the IO1 reactant were found at $1757 / 1752 / 1747,1671,1472,1191,1124,818,747$ and $636 \mathrm{~cm}^{-1}$. The bands due to the IO2 product were detected at 1750, 1680, $1675,1463 / 1459,1416,1390,1276 / 1275 / 1271,1185,1057$, $780 / 779,751,686$ and $584 \mathrm{~cm}^{-1}$. Representative examples of IR bands belonging to these two sets are shown in Fig. 1 .

Note also that upon irradiation at 314-312 $\mathrm{nm}$ the IO1 substrate could not be totally converted into the IO2 product; irradiations at these wavelengths were leading always to photostationary states. At any of such photostationary states, the population ratio of $\mathbf{I O 1}$ and $\mathbf{I O 2}$ was found to be dependent on the wavelength of UV light used for irradiation. The changes in the relative populations of IO1 and IO2 were more pronounced when irradiation was performed at wavelengths shorter than $312 \mathrm{~nm}$. However, exposure of the matrix to UV $(\lambda \leq 311 \mathrm{~nm}$ ) light was inducing (together with IO1 $\leftrightarrow$ IO2 isomerization) other photoprocesses, which consume the amino-oxo AO tautomer.

\subsection{Phototautomeric reactions consuming the amino-oxo form}

The spectral signatures of minor IO1 and IO2 forms, identified on the basis of UV (314-312 nm) irradiation, are all of low or very low intensity. The IR bands due to the significantly more populated AH1, AH2 and AO forms are (nearly all) much stronger. The strongest in the whole IR spectrum are the 
bands due to AH1 and AH2. As it has been recently reported, ${ }^{9}$ NIR irradiation of matrix-isolated cytosine at $7013 \mathrm{~cm}^{-1}$ causes the AH1 $\rightarrow \mathbf{A H 2}$ rotamerization, whereas NIR irradiation at $7034 \mathrm{~cm}^{-1}$ results in the opposite $\mathbf{A H 2} \rightarrow \mathbf{A H 1}$ conversion. Then the spectral changes induced by such NIR irradiations (see Fig. S1a and S2a in the ESI $\dagger$ ) serve as an effective tool for identification of the bands due to AH1 and AH2 forms in the spectrum of matrix-isolated cytosine. The bands assigned in the previous section of the current paper to IO1 and IO2 are not affected by NIR irradiation at either $7013 \mathrm{~cm}^{-1}$ or $7034 \mathrm{~cm}^{-1}$. Apart from the bands attributed to IO1 and IO2, another set of bands, with the most intense features at $3471,1720 / 1717,1656,1538$ and $1474 \mathrm{~cm}^{-1}$, remained unchanged upon any NIR irradiation (see Fig. S1 and S2 (ESI $\dagger$ ), traces $a$ and $b$ ). These bands are the spectral signatures of the aminooxo AO tautomer.

UV irradiations at 312.0 and at $311.5 \mathrm{~nm}$, similarly to irradiations at longer wavelengths, induced no changes in cytosine molecules adopting the AO form. On the other hand, irradiations at shorter wavelengths (starting from 311.2 and $311.0 \mathrm{~nm}$ ) led to photoprocesses consuming the AO form. Upon irradiation at $311 \mathrm{~nm}$, the population of AO systematically decreases (see Fig. 2a), as it is indicated by the intensity decrease of the characteristic bands due to $\mathbf{A O}$ form, e.g. those due to the stretching vibrations of the $\mathrm{N} 1-\mathrm{H}$ and $\mathrm{C}=\mathrm{O}$ groups (found at 3471 and at $1720 / 1717 \mathrm{~cm}^{-1}$, respectively, see Fig. 3). The decrease of the bands assigned to AO is accompanied by increase of many other bands in the IR spectrum (Fig. 2a).

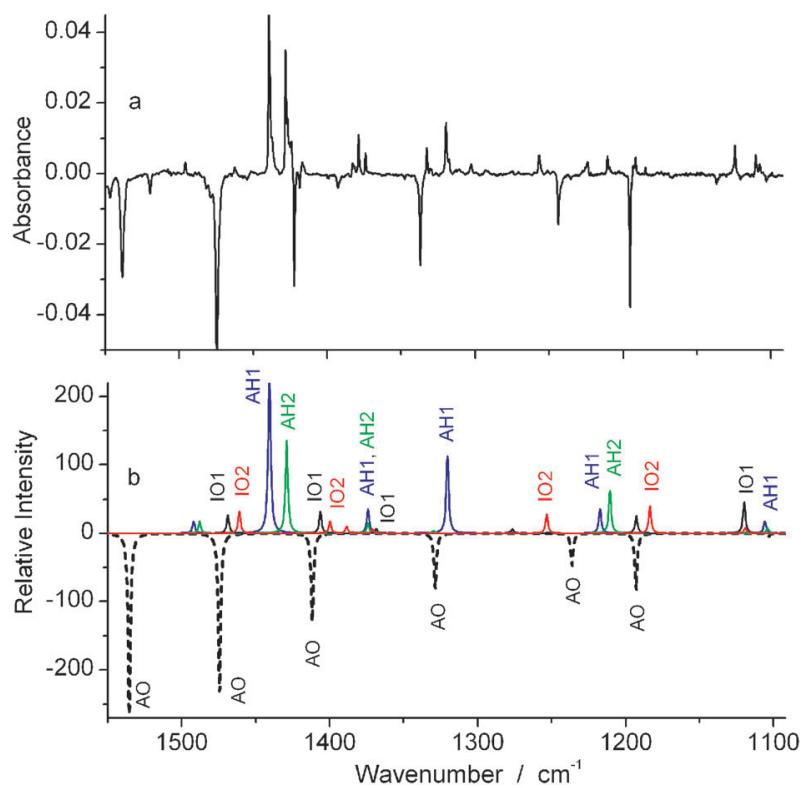

Fig. 2 Phototransformation of the AO tautomer of matrix-isolated cytosine into the AH1, AH2, IO1 and IO2 forms. (a) Subtraction result: the experimental spectrum recorded after UV irradiation at $311 \mathrm{~nm}$ minus the spectrum recorded after the previous UV irradiation at $312 \mathrm{~nm}$; (b) the spectra theoretically simulated at the DFT(B3LYP)/ 6-31++G(d,p) level for AH1 (blue), AH2 (green), IO1 (black solid line), IO2 (red) and AO (black dashed line) isomers of cytosine. Intensities in the theoretical spectra have the following weights: 2 for AH1, 1 for AH2, 1 for IO1, 1 for IO2 and -5 for AO. Theoretical wavenumbers were scaled by 0.978 .
Comparison with the spectra simulated for AH1, AH2, IO1 and IO2 (Fig. 2b) suggests that populations of all of these four isomers increase at the expense of the consumed AO form.

As far as the imino-oxo IO1 and IO2 forms are concerned, their photostationary ratio obtained after irradiation at $311 \mathrm{~nm}$ was, in a good approximation, the same as that obtained after irradiation at $312 \mathrm{~nm}$ (Fig. 3). A shift of the wavelength of the exciting UV light by only $1 \mathrm{~nm}$ did not noticeably change this photostationary ratio. However, such a small wavelength change had a dramatic effect on the photochemical behavior of $\mathbf{A O}$ form. Upon irradiation at $312 \mathrm{~nm}$ the population of $\mathbf{A O}$ stayed unchanged, whereas by irradiation at $311 \mathrm{~nm}$ this form was quickly consumed and transformed into photoproducts (see the decreasing bands at 3471, at $1720 / 1717$ and at $743 \mathrm{~cm}^{-1}$ in Fig. 3). Comparison of the two spectra presented in Fig. 3 demonstrates that upon $\operatorname{UV}(\lambda=311 \mathrm{~nm})$ irradiation the population decrease of $\mathbf{A O}$ was accompanied by an increase of population of both imino-oxo IO1 and IO2 isomers. It is revealed by the intensity increase of the IR bands at 1757 , 1752,1747 and $747 \mathrm{~cm}^{-1}$ (all assigned to IO1) as well as by the intensity increase of the bands at 1750 and $751 \mathrm{~cm}^{-1}$ (assigned to IO2). Hence, the total population of the imino-oxo tautomer (in its IO1 and IO2 forms) must have increased at the expense of the amino-oxo AO tautomer. This proves the occurrence of the amino $\rightarrow$ imino photoisomerization in cytosine monomers isolated in low-temperature environment and excited with UV $(\lambda \leq 311 \mathrm{~nm})$ light. The photochemical behavior of the band

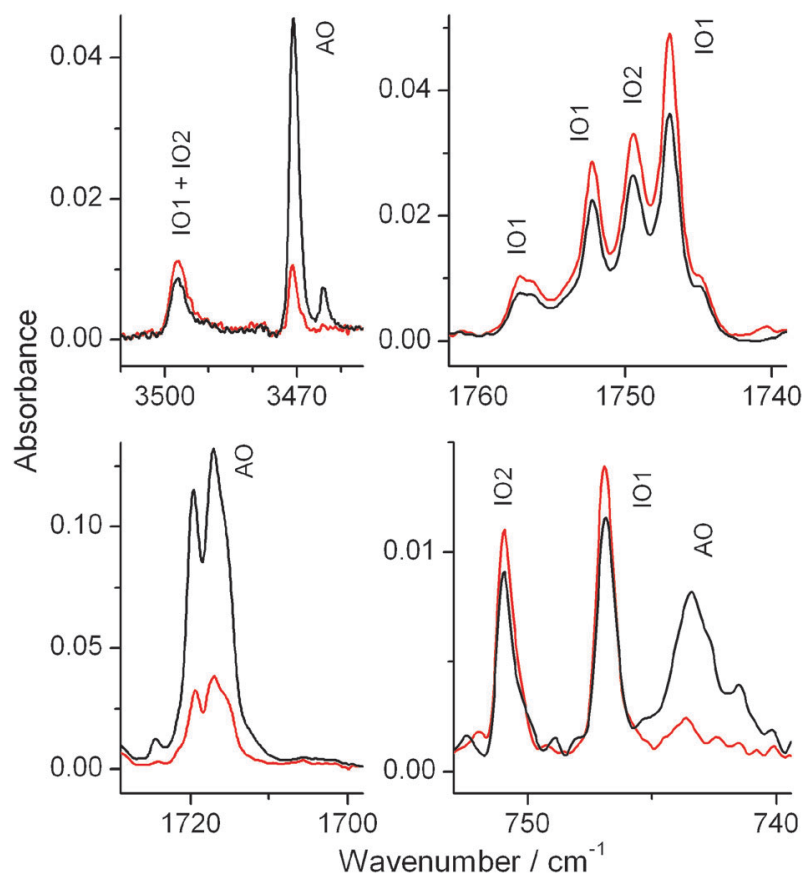

Fig. 3 Spectral indications of the amino $\rightarrow$ imino phototautomeric reaction converting the AO tautomer into IO1 and IO2 forms. Fragments of the infrared spectrum of cytosine monomers isolated in an Ar matrix: (black) recorded after irradiation of the matrix with $\mathrm{UV}(\lambda=312 \mathrm{~nm})$ monochromatic laser light; (red) recorded after subsequent irradiation of the matrix with UV $(\lambda=311 \mathrm{~nm})$ monochromatic laser light. The bands marked as IO1 and IO2 were attributed to these forms on the basis of their behavior upon irradiation at $312 \mathrm{~nm}$ (see Fig. 1). 
observed at $3497 \mathrm{~cm}^{-1}$ provides a further support to this conclusion. A counterpart of this band was observed ${ }^{8}$ at $3500 \mathrm{~cm}^{-1}$ in the spectrum of cytosine monomers in helium nanodroplets. This absorption was interpreted ${ }^{8}$ as a result of overlapping bands due to the N1H stretching vibrations in both IO1 and IO2 forms. According to the theoretical calculations, both bands should be placed at very similar wavenumbers and have very similar absolute intensities. Hence, the increase of the IR absorption at $3497 \mathrm{~cm}^{-1}$ (see Fig. 3), in the spectrum of cytosine isolated in an Ar matrix and irradiated with UV $(\lambda=311 \mathrm{~nm})$ light, must reflect increase of the combined populations of IO1 and IO2 forms.

$\mathrm{UV}$-induced consumption of $\mathbf{A O}$ form and the corresponding population increase of the photogenerated products were found to be basically the same for irradiations at any wavelength in the $311-300 \mathrm{~nm}$ range. Only the photostationary population ratio of IO1 and IO2 showed a non-negligible dependence on the wavelength of the exciting light. Intensity decrease and ultimate disappearance of the bands due to AO, occurring upon irradiation at 311-300 nm, allowed an easy identification of the IR spectral features of this form. In the $1800-1000 \mathrm{~cm}^{-1}$ range, shown in Fig. 2 and 4, the spectrum of the bands decreasing upon UV $(\lambda=311-300 \mathrm{~nm})$ irradiation agrees well with the spectrum theoretically predicted for the AO tautomer. Population increase of the amino-hydroxy AH1 and $\mathbf{A H 2}$ forms accompanied consumption of the AO tautomer. This oxo $\rightarrow$ hydroxy phototautomerism occurred upon UV irradiation at $311 \mathrm{~nm}$ (Fig. 2) as well as upon irradiation at shorter wavelengths down to $300 \mathrm{~nm}$ (Fig. 5 and Fig. S1-S3 in the ESI $\dagger$ ). All bands due to AH1 and AH2 (experimentally identified by the effects of NIR irradiation, Fig. S1 and S2 $(\mathrm{ESI} \dagger))$ increased at the expense of the $\mathbf{A O}$ reactant. The increasing spectrum included the most characteristic bands due to $\mathrm{OH}$ stretching vibrations, observed at 3601 and $3591 \mathrm{~cm}^{-1}$ (see Fig. 5 and Fig. S1 and S3 (ESI $\dagger$ )). In the previous studies of Szczesniak et al. ${ }^{10}$ where cytosine was irradiated at much shorter wavelengths $(\lambda>250 \mathrm{~nm})$, no growth but small decrease of the bands due to the aminohydroxy tautomer was reported, with simultaneous significant reduction of the intensity of the bands due to the aminooxo form.

The photoeffects described above allowed attribution of the bands observed in the IR spectrum to particular forms of cytosine. The complete list of assignments of the IR absorption bands of matrix-isolated cytosine monomers to the spectra of AO, AH1, AH2, IO1 and $\mathbf{I O 2}$ isomers is provided in Table S1 in the ESI $\dagger$ (see also Fig. S1, S2 and S4 in the ESI $\dagger$ ).

Population increase of AH1, AH2, IO1 and IO2 forms, occurring (upon irradiations at 311-300 nm) at the expense of the decreasing population of AO (see Fig. 2, 3 and 5 and Fig. S1-S3 in the ESI $\dagger$ ), does not mean that all the four isomers are primary photoproducts generated directly from the AO reactant. Most probably, the UV-induced oxo $\rightarrow$ hydroxy and amino $\rightarrow$ imino hydrogen-atom-transfer processes generate AH1 and IO1 as primary products (see Scheme 3). Forms AH1 and $\mathbf{I O 1}$ can then be partially converted into their $\mathbf{A H 2}$ and IO2 counterparts. The AH1 $\leftrightarrow$ AH2 equilibrium can be achieved during vibrational relaxation following the UV-induced hydrogen-atom transfer. It can be also induced by exposure of the matrix to NIR radiation of the spectrometer source. As described in the previous section, UV excitation, causing the syn $\leftrightarrow$ anti isomerization, should be the driving force of the IO1 $\leftrightarrow$ IO2 photoequilibrium. In such a manner, as shown in Scheme 3, forms AH2 and $\mathbf{I O 2}$ are also populated, though not directly, at the expense of the decreasing population of AO.

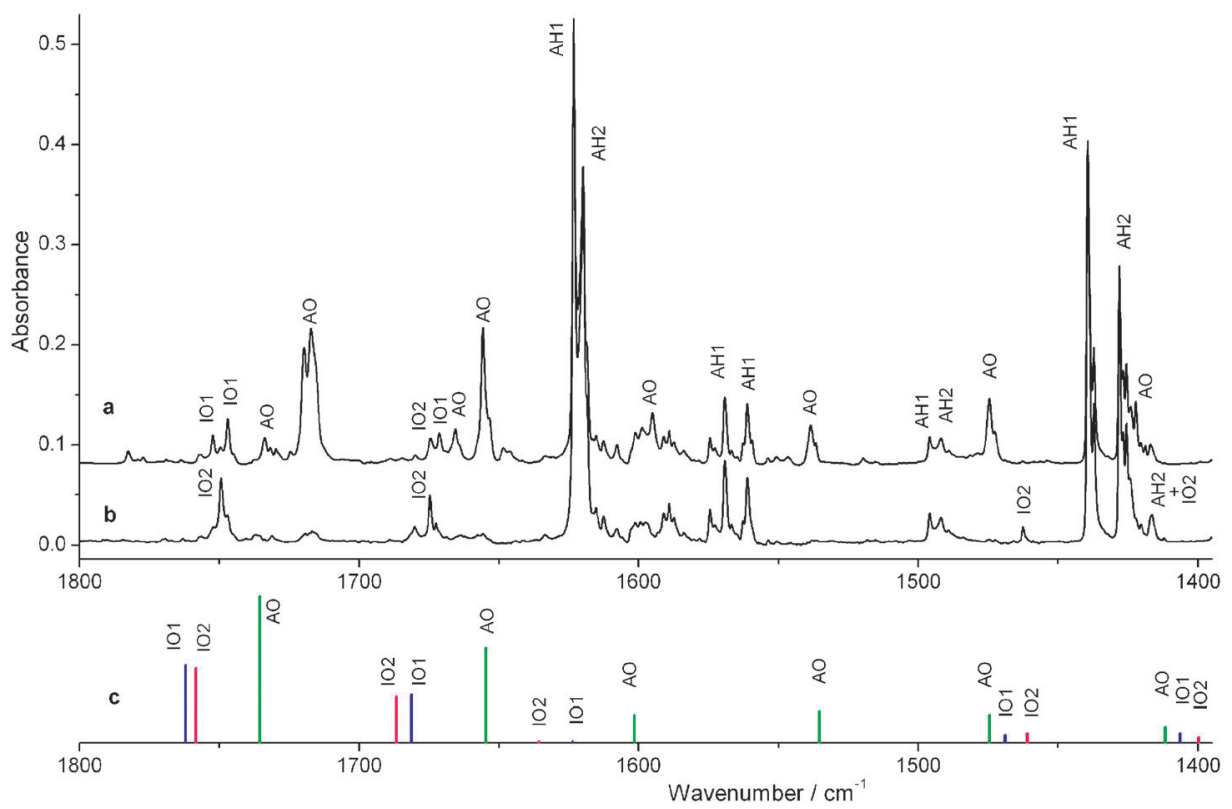

Fig. 4 Fragments of the infrared spectrum of cytosine monomers isolated in an Ar matrix: (a) recorded after deposition of the matrix; (b) recorded after irradiation of the matrix with $\mathrm{UV}(\lambda=300 \mathrm{~nm})$ monochromatic laser light; (c) calculated at the DFT(B3LYP)/6-31++G(d,p) level for: (green) AO, (blue) IO1 and (magenta) IO2 forms of cytosine. In the theoretical spectra wavenumbers were scaled by 0.978 , and intensities of IO1 and IO2 were scaled by 0.5. The bands marked as AH1 and AH2 were attributed to these forms on the basis of their behavior upon NIR irradiations at $7034 \mathrm{~cm}^{-1}$ and at $7013 \mathrm{~cm}^{-1}$ (see ref. 9 and Fig. S2 in the ESI $\dagger$ ). 


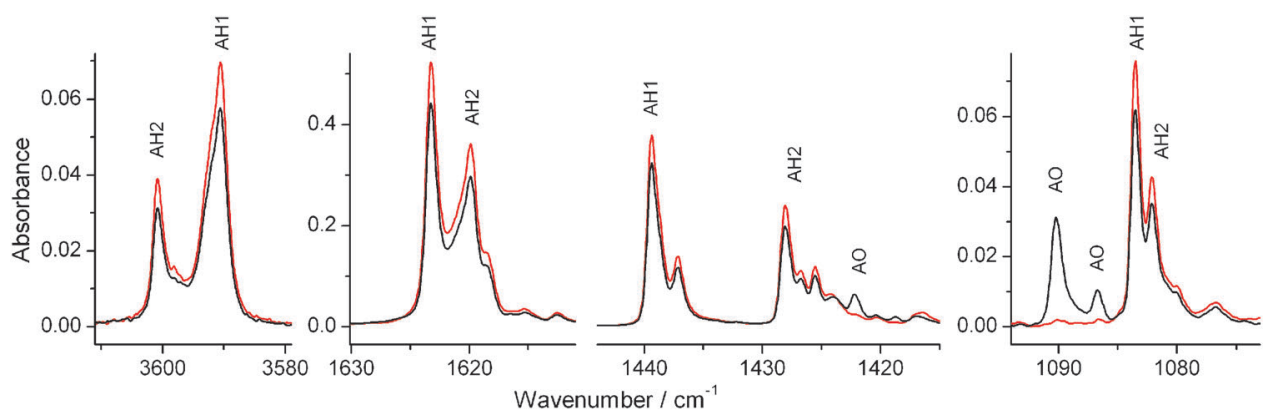

Fig. 5 Spectral indications of the oxo $\rightarrow$ hydroxy phototautomeric reaction converting the AO tautomer into AH1 and AH2 forms. Fragments of the infrared spectrum of cytosine monomers isolated in an Ar matrix: (black) recorded after deposition of the matrix; (red) recorded after irradiation of the matrix with UV $(\lambda=300 \mathrm{~nm})$ monochromatic laser light. The bands marked as AH1 and AH2 were attributed to these forms on the basis of their behavior upon NIR irradiations at $7034 \mathrm{~cm}^{-1}$ and at $7013 \mathrm{~cm}^{-1}$ (see ref. 9 and Fig. S1 and S2 in the ESI $\dagger$ ).

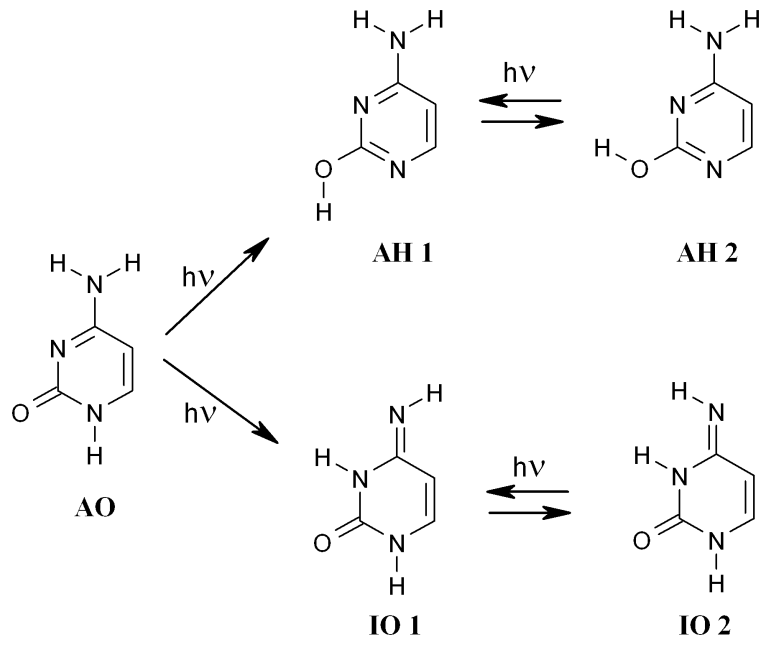

Scheme 3 UV-induced hydrogen-atom-transfer processes converting the amino-oxo tautomer of cytosine into the amino-hydroxy and imino-oxo forms.

\subsection{More on the $s y n \leftrightarrow$ anti photoisomerization in the imino-oxo forms}

Upon irradiations of the matrix at wavelengths shorter than $311 \mathrm{~nm}$, the population ratio of IO1 and IO2 was changing. The relative population of $\mathbf{I O 2}$ increased upon decreasing the wavelength of the exciting UV light. At the photostationary state resulting after irradiation at $300 \mathrm{~nm}$ (Fig. 6, red trace, see also Fig. S5 in the ESI $\dagger$ ), nearly all the population of the imino-oxo tautomer was converted into IO2.

Following the irradiation at $300 \mathrm{~nm}$, the matrix was exposed again to longer-wavelength $\mathrm{UV}(\lambda=311 \mathrm{~nm})$ light. This last irradiation shifted the ratio of IO1 and IO2 to a state with higher population of IO1 and lower population of IO2, with respect to the photoequilibrium previously induced by irradiation at $300 \mathrm{~nm}$ (see Fig. 6, blue trace). Observation of the partial repopulation of the IO1 isomer, nearly totally consumed by the previous shorter-wavelength irradiation, provides a direct proof of the photoreversibility of the syn $\leftrightarrow$ anti photoisomerization involving the two imino-oxo forms of cytosine.

A difference spectrum, obtained by subtraction of the spectrum recorded after $300 \mathrm{~nm}$ irradiation from the spectrum recorded after the subsequent irradiation at $311 \mathrm{~nm}$, is presented

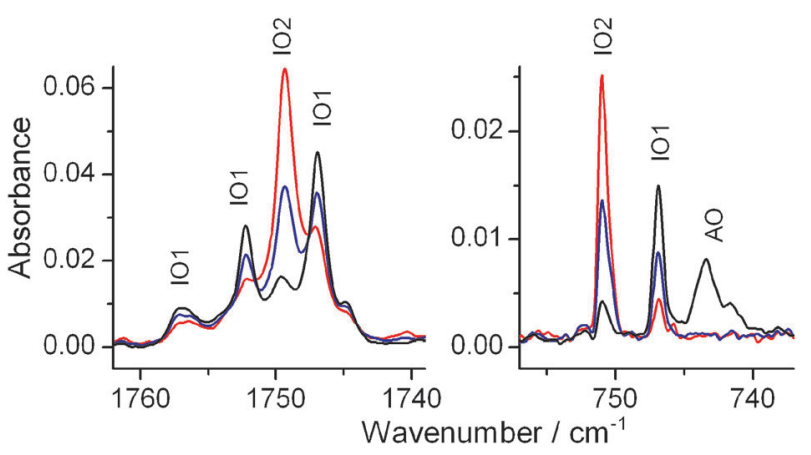

Fig. 6 Photostationary states of the IO1 $\leftrightarrow$ IO2 conversion as a function of the wavelength of exciting UV light. Fragments of the infrared spectrum of cytosine monomers isolated in an Ar matrix: (black) recorded after deposition of the matrix; (red) recorded after irradiation of the matrix with $\mathrm{UV}(\lambda=300 \mathrm{~nm})$ monochromatic laser light; (blue) recorded after subsequent irradiation of the matrix with $\mathrm{UV}(\lambda=311 \mathrm{~nm})$ monochromatic laser light.

in Fig. 7. Since the $300 \mathrm{~nm}$ irradiation led to total consumption of AO (see Fig. 4-6 and Fig. S1 and S2 in the ESI $\dagger$ ) and since AH1 and AH2 do not absorb UV light at either $300 \mathrm{~nm}$ or $311 \mathrm{~nm}$, the spectral changes induced by the last $311 \mathrm{~nm}$ irradiation must concern only the IO1 and IO2 imino-oxo isomers. The spectrum of the bands increasing upon the last irradiation at $311 \mathrm{~nm}$ and the spectrum of the bands decreasing upon this irradiation are compared (in Fig. 7) with the spectra theoretically simulated for IO1 and IO2. The good agreement between the experimental and theoretical spectra supports the correctness of the assignment of these two sets of bands to IO1 and IO2. Moreover, this comparison supports the assignment of the imino-oxo form more populated in the matrix before any irradiation to IO1, as well as the assignment of the form being only very slightly populated just after deposition of the matrix to IO2. Such interpretation fully agrees with the theoretical predictions of the relative energies of the isomeric forms of cytosine. 5,16

\subsection{Minor photoreactions}

The main photoeffect observed upon UV irradiation of matrixisolated cytosine concerned the photoreactions consuming AO tautomeric form. There is no doubt that the majority of $\mathbf{A O}$ 

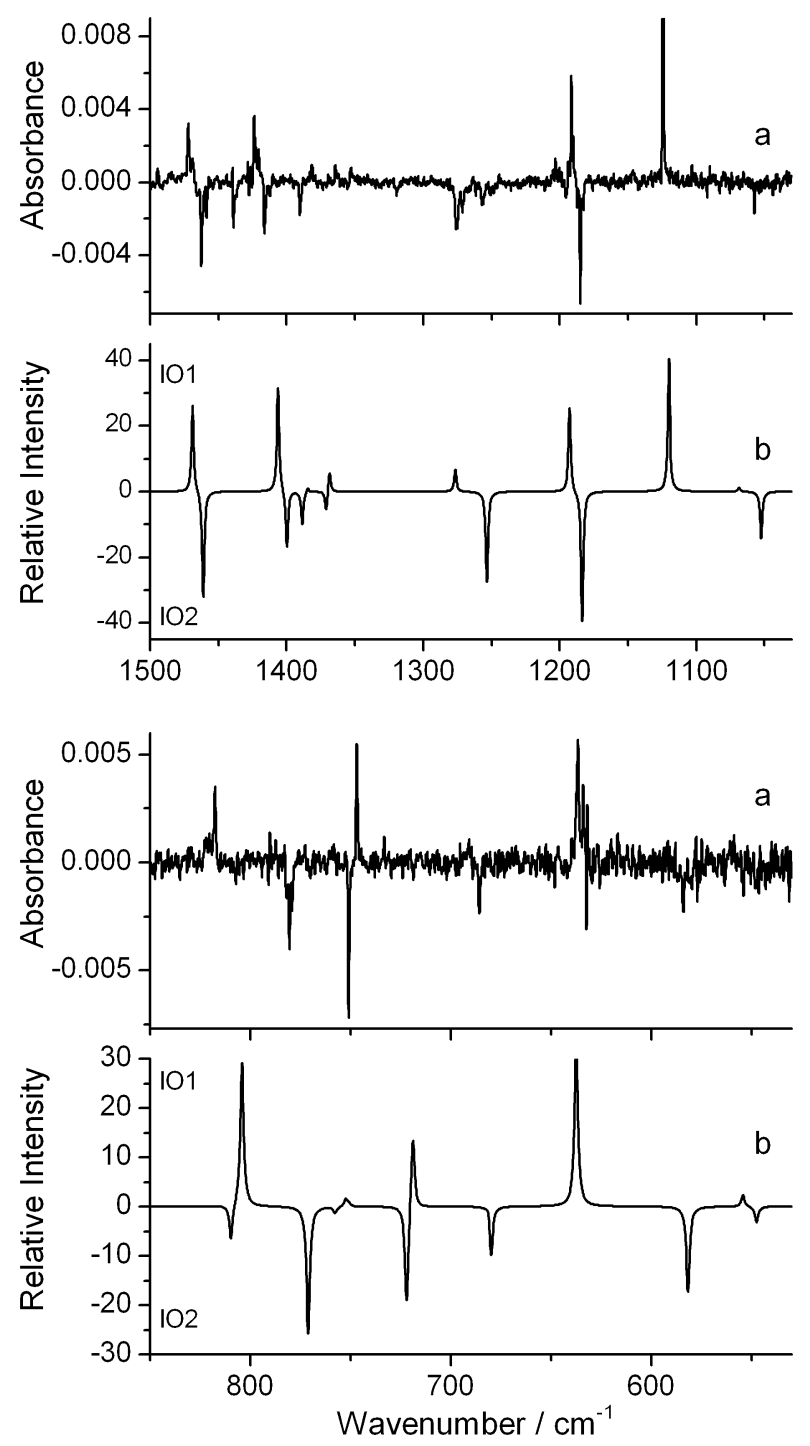

Fig. 7 Identification of IO1 and IO2 imino-oxo isomers of cytosine: (a) difference spectrum, obtained by subtraction of the spectrum recorded after $\mathrm{UV}(\lambda=300 \mathrm{~nm})$ irradiation from the spectrum recorded after the subsequent $\mathrm{UV}(\lambda=311 \mathrm{~nm})$ irradiation; (b) subtraction result: the spectrum theoretically simulated for the IO1 form minus the spectrum simulated for IO2. Theoretical spectra were calculated at the DFT(B3LYP)//6-31++G(d,p) level and the theoretical wavenumbers were scaled by 0.978 .

population was transformed, by the oxo $\rightarrow$ hydroxy and amino $\rightarrow$ imino phototautomeric reactions, into AH and IO tautomers. However, irradiation of the matrix with UV ( $\lambda=311-300 \mathrm{~nm}$ ) light, causing decrease of the population of the AO reactant, led also to the appearance of a structured IR absorption at 2300-2230 $\mathrm{cm}^{-1}$ (Fig. 8). The main component of this complex band was found at $2266 \mathrm{~cm}^{-1}$. Most probably, this band indicates that an open-ring isocyanate product was generated in an $\alpha$-bond cleavage photoreaction. Analogous processes were previously observed for matrix-isolated 1-methylcytosine and the model compound 1-methyl-2(1H)-pyrimidinone. ${ }^{18}$ The structures of these compounds are very similar to that of the AO tautomer of cytosine. For 1-methylcytosine and for 1-methyl-2(1H)-pyrimidinone, UV irradiation caused generation

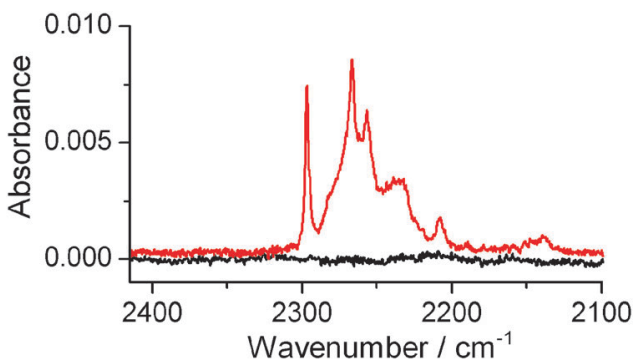

Fig. 8 Fragment of the infrared spectrum of cytosine monomers isolated in an Ar matrix: (black) recorded after deposition of the matrix; (red) recorded after irradiation of the matrix with UV $(\lambda=300 \mathrm{~nm})$ monochromatic laser light.

of photoproducts characterized by strong, structured IR absorptions centered at 2261 and $2262 \mathrm{~cm}^{-1}$, respectively. Such frequency and high intensity is typical of bands due to the "antisymmetric" stretching vibration of the $-\mathrm{N}=\mathrm{C}=\mathrm{O}$ isocyanate group. For instance, in the IR spectrum of methylisocyanate, ${ }^{19}$ a very intense, complex absorption (with a principal component at $2289 \mathrm{~cm}^{-1}$ ) was observed in the $2360-2235 \mathrm{~cm}^{-1}$ region. Detailed analysis, carried out for the photoproduct generated upon UV irradiation of 1-methyl2(1H)-pyrimidinone, ${ }^{18}$ confirmed the open-ring, conjugated isocyanate structure of this species. Hence, it looks quite likely that also for the AO isomer of cytosine, UV excitation generates the open-ring isocyanate as a minor photoproduct. The amount of this product photogenerated from cytosine must be really tiny, because the observed intensity of the spectral signature at $c a .2266 \mathrm{~cm}^{-1}$ was low and absolute intensities of IR bands due to "antisymmetric" stretching vibrations of the $-\mathrm{N}=\mathrm{C}=\mathrm{O}$ isocyanate groups are very high, ${ }^{18,19}$ usually higher than $1000 \mathrm{~km} \mathrm{~mol}^{-1}$.

\subsection{Remarks on the mechanisms of the observed photoisomerizations}

The syn $\leftrightarrow$ anti photoisomerization, observed for IO1 and IO2 forms of cytosine, belongs to the known category of photoinduced rotations around $\mathrm{C}=\mathrm{N}$ double bonds. The mechanism of such photoisomerization is similar to that of the photoinduced cis $\leftrightarrow$ trans rotation around $\mathrm{C}=\mathrm{C}$ double bonds. In such photoreactions, the key role is played by a lowenergy conical intersection between $S_{1}$ and $S_{0}$, placed at a structure with the substituent group (attached to $\mathrm{C}=\mathrm{N}$ ) rotated by $\mathrm{ca} .90^{\circ}$, with respect to the ground-state minima of the syn and anti forms. ${ }^{20,21}$ The shapes of the potential energy surfaces of $S_{1}$ and $S_{0}$ determine the typical photochemical behavior of the compounds with $\mathrm{C}=\mathrm{N}$ groups. Upon UV excitation, such compounds undergo a syn $\leftrightarrow$ anti photoisomerization leading to a photostationary state. The relative populations of syn and anti isomers at a photostationary state are the function of a wavelength of UV light used for excitation. ${ }^{21}$ Then, the photochemical behavior, observed in the current work for IO1 and IO2 isomers of cytosine, follows the typical characteristics of syn $\leftrightarrow$ anti photoisomerizations in compounds with exocyclic $\mathrm{C}=\mathrm{N}$ groups: (i) UV irradiation led to conversion of IO1 and IO2 
forms into each other; (ii) prolonged irradiation at each excitation wavelength was leading to a photostationary state; (iii) at each of the observed photostationary states, the population ratio of IO1 and IO2 was a function of the applied UV wavelength.

The phototransformation of the amino-oxo AO form into the amino-hydroxy AH tautomer is similar to the oxo $\rightarrow$ hydroxy phototautomerism, previously observed for matrixisolated heterocyclic compounds such as 4(3H)-pyrimidinone or 2(1H)-pyridinone. ${ }^{22}$ Photoinduced dissociation of the $\mathrm{N}-\mathrm{H}$ bond, on the hypersurface of a repulsive $\pi \sigma^{*}$ state, was proposed to be a key step of the PIDA (PhotoInduced Dissociation Association) mechanism of such phototautomeric reactions. ${ }^{23}$ Hence, the oxo $\rightarrow$ hydroxy phototautomerism in isolated heterocyclic molecules may be treated as a case example of photochemistry on surfaces of dissociative $\pi \sigma^{*}$ states. $^{24}$ The $\mathbf{A O} \rightarrow \mathbf{A H}$ phototransformation in cytosine seems to belong to this class of UV-induced processes.

Also the amino $\rightarrow$ imino phototautomeric reaction converting AO form into the IO tautomer can be treated as a photoinduced dissociation-association (PIDA) process. Recent experiments, carried out by King et al., ${ }^{25}$ demonstrated that a hydrogen atom of the $\mathrm{NH}_{2}$ group in UV-excited aniline dissociates on the potential energy surface of a repulsive $\pi \sigma^{*}$ state. Most probably, processes of this type are responsible for the amino $\rightarrow$ imino phototautomerism found by Akai et al. $^{26}$ in matrix-isolated 2-aminopyridine and its methylated derivatives. By analogy, it looks very likely that the AO $\rightarrow$ IO phototransformation in cytosine is also governed by the PIDA mechanism, involving a key role of dissociative $\pi \sigma^{*}$ states.

\section{Conclusions}

Experiments on cytosine monomers isolated in Ar matrices and irradiated with monochromatic UV laser light allowed identification of the amino-hydroxy, amino-oxo, and two imino-oxo isomers of the compound. The two iminooxo forms of cytosine were unambiguously experimentally observed for the first time. These results were combined with separation of the IR spectral signatures of the two aminohydroxy conformers, possible thanks to the effect of monochromatic NIR irradiation of matrix-isolated cytosine. Altogether, five isomeric forms were experimentally found for cytosine monomers trapped in low-temperature matrices. Four different photoinduced transformations of isomeric forms of cytosine were observed (Scheme 3): (i) UV-induced syn $\leftrightarrow$ anti mutual isomerizations of the two imino-oxo forms; (ii) NIR-induced conformational isomerization of the two amino-hydroxy forms; (iii) UV-induced oxo $\rightarrow$ hydroxy phototautomeric reaction; (iv) UV-induced amino $\rightarrow$ imino phototautomeric reaction. Photoreactions (i), (iii) and (iv) were induced by irradiation of cytosine monomers with 314-300 nm UV light. Besides providing experimental proofs of the presence of all five isomers of cytosine in lowtemperature matrices, the observed photoreactions allowed also a reliable assignment of the IR bands observed in the experimentally recorded spectra to particular isomeric forms of cytosine.

\section{Acknowledgements}

The research leading to these results has received funding from the European Community's Seventh Framework Programme under grant agreement no 228334.

\section{References}

1 C. Canuel, M. Mons, F. Piuzzi, B. Tardivel, I. Dimicoli and M. Elhanine, J. Chem. Phys., 2005, 122, 074316.

2 H. Kang, K. T. Lee, B. Jung, Y. J. Ko and S. K. Kim, J. Am. Chem. Soc., 2002, 124, 12958-12959; S. Ullrich, T. Schultz, M. Z. Zgierski and A. Stolow, Phys. Chem. Chem. Phys., 2004, 6, 2796-2801; K. Kosma, Ch. Schröter, E. Samoylova, I. V. Hertel and T. Schultz, J. Am. Chem. Soc., 2009, 131, 16939-16943; T. Gustavsson, R. Improta and D. Markovitsi, J. Phys. Chem. Lett., 2010, 1, 2025-2030.

3 H. R. Hudock and T. J. Martínez, ChemPhysChem, 2008, 9, 2486-2490; M. Merchán and L. Serrano-Andrés, J. Am. Chem. Soc., 2003, 125, 8108-8109; N. Ismail, L. Blancafort, M. Olivucci, B. Kohler and M. A. Robb, J. Am. Chem. Soc., 2002, 124, 6818-6819; L. Blancafort and M. A. Robb, J. Phys. Chem. A, 2004, 108, 10609-10614.

4 G. Fogarasi, J. Phys. Chem. A, 2002, 106, 1381-1390; M. Piacenza and S. Grimme, J. Comput. Chem., 2004, 25, 83-98; J. K. Wolken, Ch. Yao, F. Tureček, M. J. Polce and Ch. Wesdemiotis, Int. J. Mass Spectrom., 2007, 267, 30-42.

5 S. A. Trygubenko, T. V. Bogdan, M. Rueda, M. Orozco, F. J. Luque, J. Šponer, P. Slavíček and P. Hobza, Phys. Chem. Chem. Phys., 2002, 4, 4192-4203; R. Kobayashi, J. Phys. Chem., 1998, 102, 10813-10817.

6 R. D. Brown, P. D. Godfrey, D. McNaughton and A. P. Pierlot, J. Am. Chem. Soc., 1989, 111, 2308-2310.

7 V. Feyer, O. Plekan, R. Richter, M. Coreno, G. Vall-llosera, K. C. Prince, A. B. Trofimov, I. L. Zaytseva, T. E. Moskovskaya, E. V. Gromov and J. Schirmer, J. Phys. Chem. A, 2009, 113, 5736-5742.

8 M. Y. Choi, F. Dong and R. E. Miller, Philos. Trans. R. Soc. London, Ser. A, 2005, 363, 393-413.

9 L. Lapinski, M. J. Nowak, I. Reva, H. Rostkowska and R. Fausto, Phys. Chem. Chem. Phys., 2010, 12, 9615-9618.

10 M. Szczesniak, K. Szczepaniak, J. S. Kwiatkowski, K. KuBulat and W. B. Person, J. Am. Chem. Soc., 1988, 110, 8319-8330; M. J. Nowak, L. Lapinski and J. Fulara, Spectrochim. Acta, Part A, 1989, 45, 229-242.

11 L. Lapinski, M. J. Nowak, J. Fulara, A. Leś and L. Adamowicz, J. Phys. Chem., 1990, 94, 6555-6564.

12 A. D. Becke, Phys. Rev. A, 1988, 38, 3098-3100.

13 C. T. Lee, W. T. Yang and R. G. Parr, Phys. Rev. B: Condens. Matter, 1988, 37, 785-789.

14 M. J. Frisch, G. W. Trucks, H. B. Schlegel, G. E. Scuseria, M.A. Robb, J. R. Cheeseman, J. A. Montgomery, Jr, T. Vreven, K. N. Kudin, J. C. Burant, J. M. Millam, S. S. Iyengar, J. Tomasi, V. Barone, B. Mennucci, M. Cossi, G. Scalmani, N. Rega, G. A. Petersson, H. Nakatsuji, M. Hada, M. Ehara, K. Toyota, R. Fukuda, J. Hasegawa, M. Ishida, T. Nakajima, Y. Honda, O. Kitao, H. Nakai, M. Klene, X. Li, J. E. Knox, H. P. Hratchian, J. B. Cross, V. Bakken, C. Adamo, J. Jaramillo, R. Gomperts, R. E. Stratmann, O. Yazyev, A. J. Austin, R. Cammi, C. Pomelli, J. W. Ochterski, P. Y. Ayala, K. Morokuma, G. A. Voth, P. Salvador, J. J. Dannenberg, V. G. Zakrzewski, S. Dapprich, A. D. Daniels, M. C. Strain, O. Farkas, D. K. Malick, A. D. Rabuck, K. Raghavachari, J. B. Foresman, J. V. Ortiz, Q. Cui, A. G. Baboul, S. Clifford, J. Cioslowski, B. B. Stefanov, G. Liu, A. Liashenko, P. Piskorz, I. Komaromi, R. L. Martin, D. J. Fox, T. Keith, M. A. Al-Laham, C. Y. Peng, A. Nanayakkara, M. Challacombe, P. M. W. Gill, B. Johnson, W. Chen, M. W. Wong, C. Gonzalez and J. A. Pople, Gaussian 03, Gaussian, Inc., Wallingford CT, 2004.

15 E. Nir, Ch. Plützer, K. Kleinermanns and M. de Vries, Eur. Phys. J. D, 2002, 20, 317-329; E. Nir, M. Müller, L. I. Grace and M. S. de Vries, Chem. Phys. Lett., 2002, 355, $59-64$. 
16 G. Fogarasi, J. Mol. Struct., 1997, 413, 271-278.

17 M. Szczesniak, J. Leszczynski and W. B. Person, J. Am. Chem. Soc., 1992, 114, 2731-2733.

18 L. Lapinski, H. Rostkowska, A. Khvorostov, R. Fausto and M. J. Nowak, J. Phys. Chem. A, 2003, 107, 5913-5919.

19 I. Reva, L. Lapinski and R. Fausto, J. Mol. Struct., 2010, 976, $333-341$.

20 D. S. Ruiz, A. Cembran, M. Garavelli, M. Olivucci and W. Fuss, Photochem. Photobiol., 2002, 76, 622-633.

21 L. Lapinski, M. J. Nowak, A. L. Sobolewski and B. Kierdaszuk, J. Phys. Chem. A, 2006, 110, 5038-5046.

22 M. J. Nowak, J. Fulara and L. Lapinski, J. Mol. Struct., 1988, 175, 91-96; L. Lapinski, M. J. Nowak, A. Les and L. Adamowicz, J. Am. Chem. Soc., 1994, 116, 1461-1467; A. Gerega, L. Lapinski,
M. J. Nowak, A. Furmanchuk and J. Leszczynski, J. Phys. Chem. A, 2007, 111, 4934- 4943.

23 B. Chmura, M. F. Rode, A. L. Sobolewski, L. Lapinski and M. J. Nowak, J. Phys. Chem. A, 2008, 112, 13655-13661.

24 M. N. R. Ashfold, G. A. King, D. Murdock, M. G. D. Nix, T. A. A. Oliver and A. G. Sage, Phys. Chem. Chem. Phys., 2010, 12, 1218-1238; A. L. Sobolewski, W. Domcke, C. Dedonder-Lardeux and C. Jouvet, Phys. Chem. Chem. Phys., 2002, 4, 1093-1100.

25 G. A. King, T. A. A. Oliver and M. N. R. Ashfold, J. Chem. Phys., 2010, 132, 214307.

26 N. Akai, K. Ohno and M. Aida, Chem. Phys. Lett., 2005, 413, 306-310; N. Akai, T. Harada, K. Shin-ya, K. Ohno and M. Aida, J. Phys. Chem. A, 2006, 110, 6016-6022; N. Akai, K. Ohno and M. Aida, J. Photochem. Photobiol., A, 2007, 187, 113-118. 